\title{
Closed-loop performance analysis for economic model predictive control of time-varying systems
}

\author{
Lars Grüne and Simon Pirkelmann
}

\begin{abstract}
In this paper we study closed loop performance estimates for economic model predictive control (MPC) of timevarying systems. In order to consider general cost functionals we use the concept of overtaking optimality. Under turnpike and continuity assumptions we show that the cost of the MPC closed loop approximates the cost of an infinite horizon optimal trajectory. The theoretical results are illustrated by a numerical example.
\end{abstract}

\section{INTRODUCTION}

In most classical model predictive control (MPC) applications it is common practice to track some desired reference trajectory that is assumed to be known beforehand. This reference has to be computed offline and the task of the MPC is to stabilize the system along this prescribed trajectory. When interested in using MPC for tracking an economically optimal reference we face two difficulties. First, it is a nontrivial task to find a reference trajectory at which the system is optimally operated, the so called "optimal reference". Second, even if the optimal reference is available, tracking MPC may not yield an optimal controller, as shown, e.g., in [10].

The second issue is efficiently addressed by economic MPC, see, e.g., [2], [5], [7], [10] or [9, Chapter 8]. In this MPC variant the economic criterion of interest is incorporated directly in the MPC stage cost and thus guarantees economic efficiency in the sense of the given criterion not only on the optimal reference but also away from it. While in most economic MPC schemes the optimal reference does not enter the optimization objective, in many formulations it nevertheless enters the (terminal) constraints [1], [2], [14], [15]. In these variants, the optimal reference is still needed and thus needs to be computed. This is relatively easy to achieve if the system is optimally operated at a steady state [2], [12]. It already becomes harder in case optimal operation takes place at a periodic orbit [13], [14], [15]. In the time varying (and non-periodic) setting of this paper, the optimal reference trajectory will in general no longer be a steady state or a periodic orbit but some more general time-varying reference, which presents an even greater challenge to precompute.

In this case, economic MPC without terminal conditions [7], [13], [8] can provide a remedy. In these schemes, appropriate assumptions on the optimal control problem under

This work was supported by DFG-Grant GR 1569/16-1.

L. Grüne (lars.gruene@uni-bayreuth.de) and S. Pirkelmann (simon.pirkelmanneuni-bayreuth.de) are with the Chair of Applied Mathematics, University of Bayreuth, 95447 Bayreuth, Germany. consideration ensures that the economic MPC scheme at the same time finds the optimal reference and produces closed loop solutions which approximate infinite horizon optimal trajectories and converge to (or at least to a neighborhood of) the optimal reference.

All papers cited so far either treat MPC without timevarying system dynamics and stage costs, with the exception of [14] which allows for periodic time dependence. In contrast to this, in this paper we consider general timevarying control systems, for which the optimal reference is in general no longer a steady-state or a periodic orbit; moreover it is a priori unknown. We thus extend results for economic MPC without terminal conditions to the time varying case. We follow the approach taken in [8] in order to derive estimates on how well the cost of the MPC closed loop performs in relation to the cost of an infinite horizon optimal control sequence.

This paper is structured as follows: We first give a brief introduction to MPC. Then we introduce an optimality concept to cope with possibly infinite cost functionals. In the next part we state assumptions that are necessary to establish relations between infinite and finite horizon optimal controls. As the main result we prove an estimate for the cost along the MPC closed loop trajectory. We conclude by giving a short numerical example.

\section{Setting}

Consider the following time-varying control system

$$
x(k+1)=f(k, x(k), u(k)), \quad x(0)=x,
$$

with $f: \mathbb{N}_{0} \times X \times U \rightarrow X$ and normed spaces $X$ and $U$. In this setting $k \in \mathbb{N}_{0}$ represents a time instant, $x(k) \in X$ is the state of the system at that time and $u(k) \in U$ is the control applied to the system during the next sampling interval. Given a control sequence $u \in U^{N}$ we denote the state trajectory which results from iteratively applying (1) starting from an initial state $x \in X$ at initial time $k$ by $x_{u}(\cdot ; k, x)$. For brevity we may omit the initial time when it is clear from the context and instead write $x_{u}(\cdot, x)$.

We define $\mathbb{X}(k) \subseteq X$ to be the sets of admissible states at time $k$ and $\mathbb{U}(k, x) \subseteq U$ as the sets of admissible control values for $x \in \mathbb{X}(k)$. Let $N \in \mathbb{N}$ be the horizon length. The sets $\mathbb{U}^{N}(k, x)$ denote the admissible control sequences for initial state $x \in \mathbb{X}(k)$ up to time $k+N$, i.e. control sequences $u \in U^{N}$ that satisfy

$u(j) \in \mathbb{U}\left(k+j, x_{u}(j ; k, x)\right)$ and $x_{u}(j+1 ; k, x) \in \mathbb{X}(k+j+1)$

for all $j=0, \ldots, N-1$. 
Let $\ell: \mathbb{N}_{0} \times X \times U \rightarrow \mathbb{R}$ be the (possibly time-varying) stage cost function and consider the cost functional

$$
J_{N}(k, x, u)=\sum_{j=0}^{N-1} \ell\left(k+j, x_{u}(j ; k, x), u(j)\right) .
$$

In each step of the MPC algorithm we solve the following optimization problem

$$
\underset{u \in \mathbb{U}^{N}(k, x)}{\operatorname{minimize}} J_{N}(k, x, u) .
$$

The optimal value function corresponding to (2) is defined by

$$
V_{N}(k, x):=\inf _{u \in \mathbb{U}^{N}(k, x)} J_{N}(k, x, u) .
$$

In the following we assume that a minimizer to (2) always exists, which will be denoted by $u_{N}^{*}$, or by $u_{N, x}^{*}$ if we want to emphasize the dependence on the initial state $x$. Note that for this optimal control it holds that $V_{N}(k, x)=J_{N}\left(k, x, u_{N, x}^{*}\right)$.

The model predictive control algorithm consists of the following steps at each time instant $k=k_{0}, k_{0}+1, \ldots$ :

1) Measure the current state $x=x(k)$ of the system.

2) Solve the optimal control problem (2) in order to obtain the optimal control sequence $u_{N, x}^{*}$.

3) Apply the first element of $u_{N, x}^{*}$ as a control to the system during the next sampling period, i.e. use the feedback law $\mu_{N}(x):=u_{N, x}^{*}(0)$.

4) Set $k:=k+1$ and go to 1 .

By iteratively applying the feedback in each step, that is by setting $x(k+1)=f\left(k, x(k), \mu_{N}(x(k))\right)$, we obtain the closed loop trajectory of the system, which we will denote by $x_{\mu_{N}}(\cdot, x)$ for the initial value $x=x\left(k_{0}\right) \in \mathbb{X}\left(k_{0}\right)$. The cost of this closed loop trajectory for $L$ time steps is defined by

$$
J_{L}^{c l}\left(k, x, \mu_{N}\right)=\sum_{j=0}^{L-1} \ell\left(k+j, x_{\mu_{N}}(j, x), \mu_{N}\left(x_{\mu_{N}}(j, x)\right)\right) .
$$

\section{OPTIMALITY NOTION}

Economic model predictive control can be seen as a tool for approximating an infinite horizon optimal control problem

$$
\underset{u \in \mathbb{U}^{\infty}(k, x)}{\operatorname{minimize}} J_{\infty}(k, x, u)=\sum_{j=0}^{\infty} \ell\left(k+j, x_{u}(j ; k, x), u(j)\right)
$$

by solving a sequence of finite horizon optimal control problems. In order to make a statement about the quality of the approximation we want to study how the cost of the trajectory generated by the MPC algorithm compares to the cost of the infinite horizon optimal trajectory. There is, however, a problem when considering the cost of infinitely long trajectories of our system. Since we did not make any assumptions on the stage cost $\ell$, we do not know that the stage cost evaluated along an infinite horizon will assume a finite value, i.e. we may have $J_{\infty}(k, x, u)=\infty$ for all or $J_{\infty}(k, x, u)=-\infty$ for some trajectory pairs $\left(x_{u}, u\right)$. If this is the case an optimality criterion in the usual sense of $J_{\infty}\left(k, x, u^{*}\right) \leq J_{\infty}(k, x, u) \forall u$ is not meaningful, since we have an infinite value on both sides of the inequality. In order to address this issue we use the concept of overtaking optimality as introduced by Gale in [6] (see also [4]).

Definition 1 (Overtaking optimality): Let $x \in \mathbb{X}(k)$ and consider a control sequence $u^{*} \in \mathbb{U}^{\infty}(k, x)$ with corresponding state trajectory $x_{u^{*}}(\cdot ; k, x)$. The pair $\left(x_{u^{*}}, u^{*}\right)$ is called overtaking optimal if

$$
\begin{aligned}
& \liminf _{K \rightarrow \infty} \sum_{j=0}^{K-1} \ell\left(k+j, x_{u}(j, x), u(j)\right) \\
&-\ell\left(k+j, x_{u^{*}}(j), u^{*}(j)\right) \geq 0
\end{aligned}
$$

for all $u \in \mathbb{U}^{\infty}(k, x)$.

Similarly we define for what control and state trajectory the system (1) exhibits the best performance.

Definition 2 (Optimal operation): Let $x \in \mathbb{X}(k)$ and consider a control sequence $u^{*} \in \mathbb{U}^{\infty}(k, x)$ with corresponding state trajectory $x^{*}=x_{u^{*}}(\cdot ; k, x)$. We say the system (1) is optimally operated at $\left(x^{*}, u^{*}\right)$ if

$$
\begin{aligned}
& \liminf _{K \rightarrow \infty} \sum_{j=0}^{K-1} \ell\left(k+j, x_{u}\left(j, x^{\prime}\right), u(j)\right) \\
&-\ell\left(k+j, x^{*}(j), u^{*}(j)\right) \geq 0
\end{aligned}
$$

for all $x^{\prime} \in \mathbb{X}(k)$ and $u \in \mathbb{U}^{\infty}\left(k, x^{\prime}\right)$. In the terminology of the introduction, $\left(x^{*}, u^{*}\right)$ is the "optimal reference".

Note the difference between the two above definitions. The first definition demands that $x_{u^{*}}$ starts in the same initial state as $x_{u}$. In contrast, the initial state of $x^{*}$ is 'free' in the second definition.

In the sequel we will assume that there exists a trajectory pair denoted by $\left(x^{*}, u^{*}\right)$ at which the system is optimally operated. We will also assume that the infinite horizon optimal solution (in the sense of Definition 1) of (3) exists for each initial condition and denote it by $u_{\infty}^{*}$.

While the concept of overtaking optimality defines a meaningful optimality criterion for our setting, the corresponding infinite horizon cost functional may be unbounded. In order to avoid this issue we introduce a modified stage cost function defined in the following.

Definition 3 (Modified cost): We define the modified stage cost as

$$
\hat{\ell}(k, x(k), u(k)):=\ell(k, x(k), u(k))-\ell\left(k, x^{*}(k), u^{*}(k)\right)
$$

and the modified cost functional as

$$
\hat{J}_{N}(k, x, u):=\sum_{j=0}^{N-1} \hat{\ell}\left(k+j, x_{u}(j ; k, x), u(j)\right) .
$$

The corresponding optimal value function is given by

$$
\begin{aligned}
\hat{V}_{N}(k, x) & :=\inf _{u \in \mathbb{U}^{N}(k, x)} \hat{J}_{N}(k, x, u) \\
& =\inf _{u \in \mathbb{U}^{N}(k, x)} J_{N}(k, x, u)-J_{N}^{*}(k) \\
& =V_{N}(k, x)-J_{N}^{*}(k)
\end{aligned}
$$


with $J_{N}^{*}(k):=\sum_{j=k}^{k+N-1} \ell\left(j, x^{*}(j), u^{*}(j)\right)$.

The above definition extends to $N=\infty$, and one easily sees that for $\hat{V}_{\infty}$ the identity

$$
\hat{V}_{\infty}\left(k, x^{*}(k)\right)=0
$$

holds for all $k \in \mathbb{N}_{0}$. In addition, from Definition 2 the inequality

$$
\hat{V}_{\infty}(k, x) \geq 0
$$

follows for all $k \in \mathbb{N}$ and $x \in \mathbb{X}(k)$ (although $\hat{V}_{N}(k, x)<0$ is possible).

It should be noted that, without further assumptions, $\hat{V}_{\infty}(k, x)$ does not necessarily attain a finite value for all $x \in \mathbb{X}(k)$, but only for the special choice of $x=x^{*}(k)$, i.e. for an initial value on the optimal trajectory $x^{*}$. In order to get finiteness of $\hat{V}_{\infty}(k, x)$ for all $x \in \mathbb{X}(k)$, we will need two assumptions, namely the turnpike property and continuity of the optimal value function $\hat{V}_{\infty}$ near $x^{*}$ as introduced in the following.

\section{TURNPIKE AND CONTINUITY ASSUMPTIONS}

Assumption 1 (Turnpike in time-varying case): Consider a trajectory pair $\left(x^{*}, u^{*}\right)$ at which the system (1) is optimally operated.

a) The optimal control problem on infinite horizon with modified stage cost $\hat{\ell}$ has the time-varying turnpike property at $\left(x^{*}, u^{*}\right)$ if the following holds: There exists $\rho \in \mathcal{L}^{1}$ such that for each $k \in \mathbb{N}_{0}$, each optimal trajectory $x_{u_{\infty}^{*}}(\cdot, x)$, $x \in \mathbb{X}(k)$ and all $P \in \mathbb{N}$ there is a set $\mathcal{Q}(k, x, P, \infty) \subseteq \mathbb{N}_{0}$ with $\# \mathcal{Q}(k, x, P, \infty) \leq P$ and

$$
\left|\left(x_{u_{\infty}^{*}}(j, x), u_{\infty}^{*}(j)\right)\right|_{\left(x^{*}(k+j), u^{*}(k+j)\right)} \leq \rho(P)
$$

for all $j \in \mathbb{N}_{0}$ with $j \notin \mathcal{Q}(k, x, P, \infty)$.

b) The optimal control problem on finite horizon has the time varying turnpike property at $\left(x^{*}, u^{*}\right)$ if the following hold: There exists $\sigma \in \mathcal{L}$ such that for each $k \in \mathbb{N}_{0}$, each optimal trajectory $x_{u_{N}^{*}}(\cdot, x), x \in \mathbb{X}(k)$ and all $N, P \in \mathbb{N}$ there is a set $\mathcal{Q}(k, x, P, N) \subseteq\{0, \ldots, N\}$ with $\# \mathcal{Q}(k, x, P, N) \leq$ $P$ and

$$
\left|\left(x_{u_{N}^{*}}(j, x), u_{N}^{*}(j)\right)\right|_{\left(x^{*}(k+j), u^{*}(k+j)\right)} \leq \sigma(P)
$$

for all $j \in\{0, \ldots, N\}$ with $j \notin \mathcal{Q}(k, x, P, N)$.

The turnpike property guarantees that the open loop solutions on infinite and finite horizon are close to the optimal trajectory of the system, at least most of the time. This is illustrated in Figure 1 for the finite horizon case.

Assumption 2 (Continuity property of $\hat{V}_{N}$ and $\hat{V}_{\infty}$ ): We assume that the optimal value functions $\hat{V}_{N}$ and $\hat{V}_{\infty}$ are (approximately) continuous at $x^{*}$ in the following uniform way: for each $k \in \mathbb{N}_{0}$ there is an open ball $\mathcal{B}_{\varepsilon}\left(x^{*}(k)\right)$, $\varepsilon>0$, around $x^{*}(k)$ and a function $\gamma_{V}: \mathbb{R}_{0}^{+} \times \mathbb{R}_{0}^{+} \rightarrow \mathbb{R}_{0}^{+}$ with $\gamma_{V}(N, r) \rightarrow 0$ if $N \rightarrow \infty$ and $r \rightarrow 0$, and $\gamma_{V}(\cdot, r)$,

\footnotetext{
${ }^{1} \mathcal{L}:=\left\{\sigma: \mathbb{R}_{0}^{+} \rightarrow \mathbb{R}_{0}^{+} \mid \sigma\right.$ is continuous and strictly decreasing with $\left.\lim _{s \rightarrow \infty} \sigma(s)=0\right\}$, cf. [11]
}

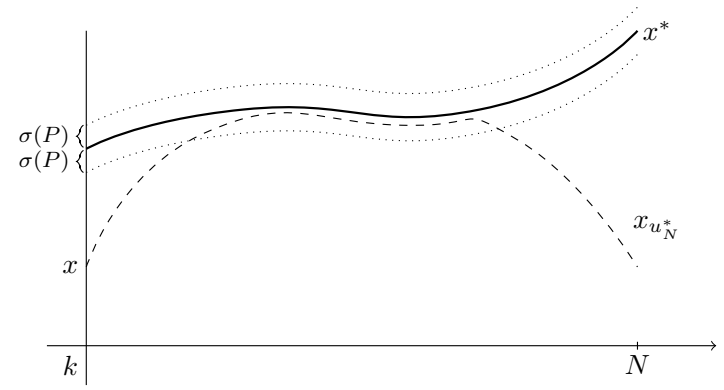

Fig. 1. Finite horizon turnpike property for time-varying systems.

$\gamma_{V}(N, \cdot)$ monotonous for fixed $r$ and $N$, such that for all $x \in \mathcal{B}_{\varepsilon}\left(x^{*}(k)\right) \cap \mathbb{X}(k)$ and all $N \in \mathbb{N} \cup\{\infty\}$ the inequality

$$
\left|\hat{V}_{N}(k, x)-\hat{V}_{N}\left(k, x^{*}(k)\right)\right| \leq \gamma_{V}\left(N,\left\|x-x^{*}(k)\right\|\right)
$$

holds, where we make the assumption that $\gamma_{V}(\infty, r)=$ : $\omega_{V}(r)$ with $\omega_{V} \in \mathcal{K}_{\infty}{ }^{2}$.

The turnpike property together with the continuity of the optimal value functions implies that $\hat{V}_{\infty}$ can only assume finite values for each $x \in X$ as shown by the following Lemma.

Lemma 1 (Finite optimal value function): Assume that the infinite horizon turnpike property from Assumption 1 a) and the continuity property from Assumption 2 hold. Then for each $k \in \mathbb{N}_{0}$ and for each $x \in X$ the value $\hat{V}_{\infty}(k, x)$ is finite.

Proof: We note that because of (6) it is sufficient to show $\hat{V}_{\infty}(k, x)<\infty$. Let $k \in \mathbb{N}_{0}$ and $x \in X$. Consider the infinite horizon optimal control sequence $u_{\infty}^{*}$. Pick $P \in \mathbb{N}$ such that $\rho(P)<\varepsilon$ with $\varepsilon$ from Assumption 2 .

Because the infinite horizon turnpike property holds we know that

$$
\left|\left(x_{u_{\infty}^{*}}(j, x), u_{\infty}^{*}(j)\right)\right|_{\left(x^{*}(k+j), u^{*}(k+j)\right)} \leq \rho(P)<\varepsilon
$$

for some $j \in \mathbb{N}_{0}$, in particular $x_{u_{\infty}^{*}}(j, x) \in \mathcal{B}_{\varepsilon}\left(x^{*}(k+j)\right)$. Thus we can apply the continuity property from Assumption 2 which yields

$$
\begin{aligned}
& \left|\hat{V}_{\infty}\left(k+j, x_{u_{\infty}^{*}}(j, x)\right)-\hat{V}_{\infty}\left(k+j, x^{*}(k+j)\right)\right| \\
& \leq \omega_{V}\left(\left\|x_{u_{\infty}^{*}}(j, x)-x^{*}(k+j)\right\|\right)<\omega_{V}(\varepsilon)
\end{aligned}
$$

where we used the monotonicity of $\omega_{V}$ in the last inequality. Because $\hat{V}_{\infty}\left(k+j, x^{*}(k+j)\right)=0$ (cf. the discussion after Definition 3) it follows that

$$
\left|\hat{V}_{\infty}\left(k+j, x_{u_{\infty}^{*}}(j, x)\right)\right|<\omega_{V}(\varepsilon) .
$$

From the optimality of $\hat{V}_{\infty}(k, x)$ it follows that

$$
\hat{V}_{\infty}(k, x) \leq \hat{J}_{j}\left(k, x, u_{\infty}^{*}\right)+\hat{V}_{\infty}\left(k+j, x_{u_{\infty}^{*}}(j, x)\right) .
$$

The term $\hat{J}_{j}\left(k, x, u_{\infty}^{*}\right)$ is finite and thus $\hat{V}_{\infty}(k, x)$ is finite, too.

\footnotetext{
${ }^{2} \mathcal{K}_{\infty}:=\left\{\alpha: \mathbb{R}_{0}^{+} \rightarrow \mathbb{R}_{0}^{+} \mid \alpha\right.$ is continuous, strictly increasing and unbounded with $\alpha(0)=0\}$
} 


\section{APPROXIMATION PROPERTIES OF THE OPTIMAL VALUE FUNCTIONS}

Now that have ensured that the optimal value function $\hat{V}_{\infty}$ is finite, we present a lemma which states that the cost of the optimal trajectory evaluated along the complete horizon is approximately the same as the cost evaluated only up to some appropriately chosen time index $K$. The result applies to both the infinite and finite horizon optimal control trajectories.

Lemma 2: a) If the system has the infinite horizon turnpike property from Assumption 1 a) and the continuity property from Assumption 2 is satisfied, then the equation

$$
\hat{V}_{\infty}(k, x)=\hat{J}_{K}\left(k, x, u_{\infty}^{*}\right)+R_{1}(k, x, K)
$$

holds with $\left|R_{1}(k, x, K)\right| \leq \omega_{V}(\rho(P))$ for all $k \in \mathbb{N}_{0}$, for all $x \in \mathbb{X}(k)$, all $N \in \mathbb{N}$, all sufficiently large $P \in \mathbb{N}$ and all $K \notin \mathcal{Q}(k, x, P, \infty)$.

b) If the system has the finite horizon turnpike property from Assumption $1 \mathrm{~b}$ ) and the continuity property from Assumption 2 is satisfied, then the equation

$$
\begin{aligned}
\hat{V}_{N}(k, x)= & \hat{J}_{K}\left(k, x, u_{N}^{*}\right) \\
& +\hat{V}_{N-K}\left(k+K, x^{*}(k+K)\right) \\
& +R_{2}(k, x, K, N)
\end{aligned}
$$

holds with $\left|R_{2}(k, x, K, N)\right| \leq \gamma_{V}(N-K, \sigma(P))$ for all $k \in \mathbb{N}_{0}$, for all $x \in \mathbb{X}(k)$, all $N \in \mathbb{N}$, all sufficiently large $P \in \mathbb{N}$ and all $K \notin \mathcal{Q}(k, x, P, N)$.

Proof: $\quad$ a) Let $k \in \mathbb{N}_{0}$ and $x \in \mathbb{X}(k)$. The dynamic programming principle (see [3]) yields

$$
\hat{V}_{\infty}(k, x)=\hat{J}_{K}\left(k, x, u_{\infty}^{*}\right)+\hat{V}_{\infty}\left(k+K, x_{u_{\infty}^{*}}(K, x)\right)
$$

for each $K \in \mathbb{N}_{0}$. This means equation (7) holds with $R_{1}(k, x, K)=\hat{V}_{\infty}\left(k+K, x_{u_{\infty}^{*}}(K, x)\right)$. Chose $P \in \mathbb{N}$ sufficiently large such that $\rho(P)<\varepsilon$ with $\rho$ from Assumption 1 a) and $\varepsilon$ from Assumption 2. Because we have $\hat{V}_{\infty}\left(k+K, x^{*}(k+K)\right)=0$ and because of the continuity of $\hat{V}_{\infty}$ we get that

$$
\begin{aligned}
& \left|R_{1}(k, x, K)\right|=\mid \hat{V}_{\infty}\left(k+K, x_{u_{\infty}^{*}}(K, x)\right) \\
& \quad-\hat{V}_{\infty}\left(k+K, x^{*}(k+K)\right) \mid \\
& \leq \omega_{V}\left(\left\|x_{u_{\infty}^{*}}(K, x)-x^{*}(k+K)\right\|\right) \\
& \leq \omega_{V}\left(\left|\left(x_{u_{\infty}^{*}}(K, x), u_{\infty}^{*}(K)\right)\right|_{\left.\left(x^{*}(k+K), u^{*}(k+K)\right)\right)}\right) \\
& \leq \omega_{V}(\rho(P)),
\end{aligned}
$$

which holds for all $K \in \mathbb{N}$ with $K \notin \mathcal{Q}(k, x, P, \infty)$ and where we used the monotonicity of $\omega_{V}$. This shows the assertion.

b) Let $k \in \mathbb{N}_{0}$ and $x \in \mathbb{X}(k)$. In the finite horizon case, the dynamic programming principle yields

$$
\hat{V}_{N}(k, x)=\hat{J}_{K}\left(k, x, u_{N}^{*}\right)+\hat{V}_{N-K}\left(k+K, x_{u_{N}^{*}}(K, x)\right)
$$

for $K \in\{0, \ldots, N\}$. Hence, (8) holds with $R_{2}(k, x, K, N)=\hat{V}_{N-K}\left(k+K, x_{u_{N}^{*}}(K, x)\right)-\hat{V}_{N-K}(k+$ $\left.K, x^{*}(k+K)\right)$. Chose $P \in \mathbb{N}$ sufficiently large such that
$\sigma(P)<\varepsilon$ holds for $\sigma$ from Assumption 1 b) and $\varepsilon$ from Assumption 2. Then we have

$$
\begin{aligned}
& \left|R_{2}(k, x, K, N)\right|=\mid \hat{V}_{N-K}\left(k+K, x_{u_{N}^{*}}(K, x)\right) \\
& \quad-\hat{V}_{N-K}\left(k+K, x^{*}(k+K)\right) \mid \\
& \leq \gamma_{V}\left(N-K,\left\|x_{u_{N}^{*}}(K, x)-x^{*}(k+K)\right\|\right) \\
& \leq \gamma_{V}\left(N-K,\left|\left(x_{u_{N}^{*}}(K, x), u_{N}^{*}(K)\right)\right|_{\left(x^{*}(K+k), u^{*}(K+k)\right)}\right)
\end{aligned}
$$

using again the monotonicity of $\gamma_{V}(N-K, \cdot)$. For $K \notin$ $\mathcal{Q}(k, x, P, N)$ it follows that $\left|R_{2}(k, x, K, N)\right| \leq \gamma_{V}(N-$ $K, \sigma(P))$ and thus the assertion.

The next lemma shows that we can exchange the infinite horizon optimal control trajectory by the one on finite horizon, at the cost of a bounded error term.

Lemma 3: If the system has the infinite and finite horizon turnpike properties from Assumption 1 and the continuity property from Assumption 2 is satisfied, then the equation

$$
\hat{J}_{K}\left(k, x, u_{\infty}^{*}\right)=\hat{J}_{K}\left(k, x, u_{N}^{*}\right)+R_{3}(k, x, K, N)
$$

holds with $\left|R_{3}(k, x, K, N)\right| \leq \gamma_{V}(N-K, \rho(P))+\gamma_{V}(N-$ $K, \sigma(P))+\omega_{V}(\sigma(P))+\omega_{V}(\rho(P))$ for all $k \in \mathbb{N}_{0}$, all $N \in \mathbb{N}$, all sufficiently large $P \in \mathbb{N}$, all $x \in \mathbb{X}(k)$ and all $K \in$ $\{0, \ldots, N\} \backslash(\mathcal{Q}(k, x, P, N) \cup \mathcal{Q}(k, x, P, \infty))$.

Proof: Consider $R_{2}(k, x, K, N)=\hat{V}_{N-K}(k+$ $\left.K, x_{u_{N}^{*}}(K, x)\right)-\hat{V}_{N-K}\left(k+K, x^{*}(k+K)\right)$ from Lemma 2 b) and define

$$
\begin{aligned}
\tilde{R}_{1}(k, x, K, N):= & \hat{V}_{N-K}\left(k+K, x_{u_{\infty}^{*}}(K, x)\right) \\
& -\hat{V}_{N-K}\left(k+K, x^{*}(k+K)\right) .
\end{aligned}
$$

Those expressions satisfy $\left|R_{2}(k, x, K, N)\right| \leq \gamma_{V}(N-$ $K, \sigma(P))$ for $K \in\{0, \ldots, N\} \backslash \mathcal{Q}(k, x, P, N)$ and $\left|\tilde{R}_{1}(k, x, K, N)\right| \leq \gamma_{V}(N-K, \rho(P))$ for $K \in \mathbb{N}_{0} \backslash$ $\mathcal{Q}(k, x, P, \infty)$ as one sees similarly to the proof of Lemma $2 \mathrm{~b})$.

The finite horizon dynamic programming principle implies that $u=u_{N}^{*}$ minimises the expression $\hat{J}_{K}(k, x, u)+$ $\hat{V}_{N-K}\left(k+K, x_{u}(K, x)\right)$, in particular we have that

$$
\begin{aligned}
& \hat{J}_{K}\left(k, x, u_{N}^{*}\right)+\hat{V}_{N-K}\left(k+K, x_{u_{N}^{*}}(K, x)\right) \\
& \leq \hat{J}_{K}\left(k, x, u_{\infty}^{*}\right)+\hat{V}_{N-K}\left(k+K, x_{u_{\infty}^{*}}(K, x)\right) .
\end{aligned}
$$

This, together with the definition of $R_{2}$ and $\tilde{R}_{1}$ implies that

$$
\begin{aligned}
& \hat{J}_{K}\left(k, x, u_{N}^{*}\right)+\hat{V}_{N-K}\left(k+K, x^{*}(k+K)\right) \\
& =\hat{J}_{K}\left(k, x, u_{N}^{*}\right)+\hat{V}_{N-K}\left(k+K, x_{u_{N}^{*}}(K, x)\right) \\
& \quad-R_{2}(k, x, K, N) \\
& \leq \hat{J}_{K}\left(k, x, u_{\infty}^{*}\right)+\hat{V}_{N-K}\left(k+K, x_{u_{\infty}^{*}}(K, x)\right) \\
& \quad-R_{2}(k, x, K, N) \\
& =\hat{J}_{K}\left(k, x, u_{\infty}^{*}\right)+\hat{V}_{N-K}\left(k+K, x^{*}(k+K)\right) \\
& \quad+\tilde{R}_{1}(k, x, K, N)-R_{2}(k, x, K, N),
\end{aligned}
$$

i.e. we have

$$
\begin{aligned}
& \hat{J}_{K}\left(k, x, u_{N}^{*}\right) \\
& \leq \hat{J}_{K}\left(k, x, u_{\infty}^{*}\right)+\tilde{R}_{1}(k, x, K, N)-R_{2}(k, x, K, N)
\end{aligned}
$$

for all $K \in\{0, \ldots, N\} \backslash(\mathcal{Q}(k, x, P, N) \cup \mathcal{Q}(k, x, P, \infty))$. 
To show the converse inequality consider $R_{1}(k, x, K)=$ $\hat{V}_{\infty}\left(k+K, x_{u_{\infty}^{*}}(K, x)\right)$ from Lemma 2 a) for which we obtained the bound $\left|R_{1}(k, x, K)\right| \leq \omega_{V}(\rho(P))$ for $K \in \mathbb{N}_{0} \backslash \mathcal{Q}(k, x, P, \infty)$, and define $\tilde{R}_{2}(k, x, K, N):=$ $\hat{V}_{\infty}\left(k, x_{u_{N}^{*}}(K, x)\right)$ for which the bound $\tilde{R}_{2}(k, x, K, N) \leq$ $\omega_{V}(\sigma(P))$ holds, given that $K \in\{0, \ldots, N\} \backslash \mathcal{Q}(k, x, P, N)$.

The infinite horizon dynamic programming principle implies

$$
\begin{aligned}
& \hat{J}_{K}\left(k, x, u_{\infty}^{*}\right)+\hat{V}_{\infty}\left(k+K, x_{u_{\infty}^{*}}(K, x)\right) \\
& \leq \hat{J}_{K}\left(k, x, u_{N}^{*}\right)+\hat{V}_{\infty}\left(k+K, x_{u_{N}^{*}}(K, x)\right)
\end{aligned}
$$

from which we get that

$$
\begin{aligned}
\hat{J}_{K}\left(k, x, u_{\infty}^{*}\right)= & \hat{J}_{K}\left(k, x, u_{\infty}^{*}\right)+\hat{V}_{\infty}\left(k+K, x_{u_{\infty}^{*}}(K, x)\right) \\
& -R_{1}(k, x, K) \\
\leq & \hat{J}_{K}\left(k, x, u_{N}^{*}\right)+\hat{V}_{\infty}\left(k+K, x_{u_{N}^{*}}(K, x)\right) \\
& \quad-R_{1}(k, x, K) \\
= & \hat{J}_{K}\left(k, x, u_{N}^{*}\right)+\tilde{R}_{2}(k, x, K, N) \\
& -R_{1}(k, x, K) .
\end{aligned}
$$

In summary we have

$$
\begin{aligned}
\hat{J}_{K}\left(k, x, u_{\infty}^{*}\right) \leq & \hat{J}_{K}\left(k, x, u_{N}^{*}\right)+\tilde{R}_{2}(k, x, K, N) \\
& -R_{1}(k, x, K)
\end{aligned}
$$

for all $K \in\{0, \ldots, N\} \backslash(\mathcal{Q}(k, x, P, N) \cup \mathcal{Q}(k, x, P, \infty))$.

Combining the two inequalities (9) and (10) we obtain

$$
\begin{gathered}
\left|R_{3}(k, x, K, N, M)\right|=\left|\hat{J}_{K}\left(k, x, u_{N}^{*}\right)-\hat{J}_{K}\left(k, x, u_{M}^{*}\right)\right| \\
\leq \max \left\{\left|\tilde{R}_{1}(k, x, K, N, M)\right|+\left|R_{2}(k, x, K, N)\right|,\right. \\
\left.\left|\tilde{R}_{2}(k, x, K, M, N)\right|+\left|R_{1}(k, x, K, M)\right|\right\} \\
=\max \left\{\gamma_{V}(N-K, \rho(P))+\gamma_{V}(N-K, \sigma(P)),\right. \\
\left.\omega_{V}(\sigma(P))+\omega_{V}(\rho(P))\right\} \\
\leq \gamma_{V}(N-K, \rho(P))+\gamma_{V}(N-K, \sigma(P)) \\
+\omega_{V}(\sigma(P))+\omega_{V}(\rho(P))
\end{gathered}
$$

which concludes the proof.

\section{MAIN RESULT}

We can now state the main result of this paper which gives an estimate of how close the closed loop cost of the MPC trajectory is to the best possible cost of an infinite horizon optimal trajectory. We first state the result in the relative abstract form (11) and provide further explanation after the proof of the theorem.

Theorem 1: Let Assumption 2 and Assumption 1 hold. Then for each $k \in \mathbb{N}_{0}$, and each sufficiently large $N$, the closed loop cost satisfies

$$
\begin{aligned}
\hat{J}_{L}^{c l}\left(k, x, \mu_{N}\right) \leq & \hat{V}_{\infty}(k, x)-\hat{V}_{\infty}\left(k+L, x_{\mu_{N}}(L, x)\right) \\
& +L \delta(N)
\end{aligned}
$$

with a function $\delta \in \mathcal{L}$.

Proof: Let $k \in \mathbb{N}_{0}$. For $i \geq k$ pick $x \in \mathbb{X}(i)$ and abbreviate $x^{+}:=f\left(i, x, \mu_{N}(x)\right)$. By the dynamic programming principle, and the definition of $\mu_{N}$ we know that

$$
\hat{\ell}\left(i, x, \mu_{N}(x)\right)=\hat{V}_{N}(i, x)-\hat{V}_{N-1}\left(i+1, x^{+}\right) .
$$

Using the definition of the optimal value function and the fact that $u_{N, x}^{*}(\cdot+1)$ and $u_{N-1, x^{+}}^{*}(\cdot)$ coincide we obtain

$$
\begin{aligned}
& \hat{V}_{N}(i, x)-\hat{V}_{N-1}\left(i+1, x^{+}\right) \\
& =\hat{J}_{N}\left(i, x, u_{N, x}^{*}\right)-\hat{J}_{N-1}\left(i+1, x^{+}, u_{N-1, x^{+}}^{*}\right) \\
& =\hat{J}_{K}\left(i, x, u_{N, x}^{*}\right)-\hat{J}_{K-1}\left(i+1, x^{+}, u_{N-1, x^{+}}^{*}\right),
\end{aligned}
$$

which holds for each $K=\{1, \ldots, N\}$.

Now let $K \in\{1, \ldots, N\}$ such that $K \notin \mathcal{Q}(i, x, P, N) \cup$ $\mathcal{Q}(i, x, P, \infty)$ and $K-1 \notin \mathcal{Q}\left(i+1, x^{+}, P, N-1\right) \cup \mathcal{Q}(i+$ $\left.1, x^{+}, P, \infty\right)$. In each of the four sets there are at most $P$ elements, thus for $N>8 P$ there is at least one such $K$ with $K \leq \frac{N}{2}$, i.e. we set $P=\left\lfloor\frac{N-1}{8}\right\rfloor$ and choose $N$ sufficiently large.

This means we can apply Lemma 3 twice with $K=K$, $N=N$ and $K=K-1, N=N-1$, respectively, to conclude that

$$
\begin{aligned}
& \hat{J}_{K}\left(i, x, u_{N, x}^{*}\right)-\hat{J}_{K-1}\left(i+1, x^{+}, u_{N-1, x^{+}}^{*}\right) \\
& =\hat{J}_{K}\left(i, x, u_{\infty, x}^{*}\right)-\hat{J}_{K-1}\left(i+1, x^{+}, u_{\infty, x^{+}}^{*}\right) \\
& \quad+R_{3}(i, x, K, N)-R_{3}\left(i+1, x^{+}, K-1, N-1\right) .
\end{aligned}
$$

Proceeding further, by applying Lemma 2 a) for $K=K$ and $K=K-1$ we conclude that

$$
\begin{aligned}
& \hat{J}_{K}\left(i, x, u_{\infty, x}^{*}\right)-\hat{J}_{K-1}\left(i+1, x^{+}, u_{\infty, x^{+}}^{*}\right) \\
& =\hat{V}_{\infty}(i, x)-\hat{V}_{\infty}\left(i+1, x^{+}\right) \\
& \quad-R_{1}(i, x, K)+R_{1}\left(i+1, x^{+}, K-1\right) .
\end{aligned}
$$

In summary, we have

$\hat{\ell}\left(i, x, \mu_{N}(x)\right)=\hat{V}_{\infty}(i, x)-\hat{V}_{\infty}\left(i+1, x^{+}\right)+R_{4}(i, x, K, N)$,

with

$$
\begin{aligned}
& R_{4}(i, x, K, N)= \\
& \quad R_{3}(i, x, K, N)-R_{3}\left(i+1, x^{+}, K-1, N-1\right) \\
& \quad-R_{1}(i, x, K)+R_{1}\left(i+1, x^{+}, K-1\right) .
\end{aligned}
$$

In addition, from Lemma 3 and Lemma 2 a) we obtain the bound

$$
\begin{aligned}
\left|R_{4}(i, x, K, N)\right| \leq & 2 \gamma_{V}(N-K, \rho(P))+4 \omega_{V}(\rho(P)) \\
& +2 \gamma_{V}(N-K, \sigma(P))+2 \omega_{V}(\sigma(P)) .
\end{aligned}
$$

Recall that for $P=\left\lfloor\frac{N-1}{8}\right\rfloor$ we have $K \leq \frac{N}{2}$ and thus $N-K \geq \frac{N}{2}$. Because of the monotonicity of $\gamma_{V}$ in its first argument, we can bound the right hand side of (13) by

$$
\begin{aligned}
& \left|R_{4}(i, x, K, N, S)\right| \leq 2 \gamma_{V}\left(\left\lfloor\frac{N}{2}\right\rfloor, \rho\left(\left\lfloor\frac{N-1}{8}\right\rfloor\right)\right) \\
& \quad+2 \gamma_{V}\left(\left\lfloor\frac{N}{2}\right\rfloor, \sigma\left(\left\lfloor\frac{N-1}{8}\right\rfloor\right)\right)+2 \omega_{V}\left(\sigma\left(\left\lfloor\frac{N-1}{8}\right\rfloor\right)\right) \\
& \quad+4 \omega_{V}\left(\rho\left(\left\lfloor\frac{N-1}{8}\right\rfloor\right)\right)=: \delta(N) .
\end{aligned}
$$


Finally, note that equation (12) was shown for all $i \geq k$, which means we can apply it to $\hat{J}_{L}^{c l}\left(k, x, \mu_{N}\right)$ with $i=k+j$, $x=x_{\mu_{N}}(j, x)$, and in each summand the estimate (14) holds. This yields

$$
\begin{aligned}
& \hat{J}_{L}^{c l}\left(k, x, \mu_{N}\right)=\sum_{j=0}^{L-1} \hat{\ell}\left(k+j, x_{\mu_{N}}(j, x), \mu_{N}\left(x_{\mu_{N}}(j, x)\right)\right) \\
& =\sum_{j=0}^{L-1} \hat{V}_{\infty}\left(k+j, x_{\mu_{N}}(j, x)\right)-\hat{V}_{\infty}\left(k+j+1, x_{\mu_{N}}(j+1, x)\right) \\
& \quad+\underbrace{R_{4}\left(k+j, x_{\mu_{N}}(j, x), K, N\right)}_{\leq \delta(N)} \\
& \leq \hat{V}_{\infty}(k, x)-\hat{V}_{\infty}\left(k+L, x_{\mu_{N}}(L, x)\right)+L \delta(N)
\end{aligned}
$$

and thus the assertion.

The result from Theorem 1 tells us that on finite horizons $L$ the MPC closed-loop trajectory approximates an infinite horizon overtaking optimal trajectory. To realize this, consider a control sequence $\bar{u}$ defined by

$$
\bar{u}(j):= \begin{cases}\mu_{N}\left(x_{\mu_{N}}\left(j, x_{0}\right)\right), & j=0, \ldots, L-1 \\ u_{\infty, \tilde{x}}^{*}, & j \geq L\end{cases}
$$

where $\tilde{x}:=x_{\mu_{N}}\left(L, x_{0}\right)$. For the cost of the corresponding trajectory we get

$$
\begin{aligned}
& \hat{J}_{\infty}\left(k, x_{0}, \bar{u}\right)=\sum_{j=0}^{\infty} \hat{\ell}\left(k+j, x_{\bar{u}}\left(j, x_{0}\right), \bar{u}(j)\right) \\
& =\underbrace{\leq \hat{V}_{\infty}\left(k, x_{0}\right)-\hat{V}_{\infty}(k+L, \tilde{x})+L \delta(N)}+\hat{V}_{\infty}(k+L, \tilde{x}) \\
& \leq \hat{V}_{\infty}\left(k, x_{0}\right)+L \delta(N)
\end{aligned}
$$

Because $\hat{V}_{\infty}\left(k, x_{0}\right)=\hat{J}_{\infty}\left(k, x_{0}, u_{\infty, x_{0}}^{*}\right)$ this is equivalent to

$$
\begin{aligned}
L \delta(N) \geq & \sum_{j=0}^{\infty} \hat{\ell}\left(k+j, x_{\bar{u}}\left(j, x_{0}\right), \bar{u}(j)\right) \\
& -\sum_{j=0}^{\infty} \hat{\ell}\left(k+j, x_{u_{\infty, x_{0}}^{*}}\left(j, x_{0}\right), u_{\infty, x_{0}}^{*}(j)\right) .
\end{aligned}
$$

From this inequality and the definition of $\hat{\ell}$ it follows that

$$
\begin{aligned}
& \liminf _{K \rightarrow \infty} \sum_{j=0}^{K-1} \ell\left(k+j, x_{\bar{u}}\left(j, x_{0}\right), \bar{u}(j)\right) \\
& -\sum_{j=0}^{K-1} \ell\left(k+j, x_{u_{\infty, x_{0}}^{*}}\left(j, x_{0}\right), u_{\infty, x_{0}}^{*}(j)\right) \leq L \delta(N) .
\end{aligned}
$$

This means that in terms of the overtaking optimality criterion the initial piece of the MPC closed-loop trajectory approximates the initial piece of the optimal trajectory $x_{u_{\infty, x_{0}}^{*}}\left(\cdot, x_{0}\right)$.

A drawback of Theorem 1 is that the error term in the estimate includes the factor $L$, which means that for fixed $N$ the estimate may get arbitrarily poor as $L$ increases. This may lead to the conjecture that in the long run the MPC closed loop trajectory deteriorates. However, an analysis of the averaged cost functionals $\bar{J}_{L}(k, x, u):=\frac{1}{L} J_{L}(k, x, u)$ along the closed loop shows that this does not happen: from (11) and the fact that $\hat{V}_{\infty}\left(k+L, x_{\mu_{N}}(L, x)\right) \geq 0$ we get

$$
\bar{J}_{L}^{c l}\left(k, x, \mu_{N}\right) \leq \frac{1}{L} \hat{V}_{\infty}(k, x)+\delta(N) .
$$

Letting $L \rightarrow \infty$ and using the definition of the modified stage cost we obtain

$$
\limsup _{L \rightarrow \infty} \bar{J}_{L}^{c l}\left(k, x, \mu_{N}\right) \leq \limsup _{L \rightarrow \infty} \bar{J}_{L}^{*}(k)+\delta(N) .
$$

Assuming that the infinite horizon averages attain finite values we get

$$
\bar{J}_{\infty}^{c l}\left(k, x, \mu_{N}\right) \leq \bar{J}_{\infty}^{*}(k)+\delta(N) .
$$

This means the average cost of the MPC closed loop is approximately the same as the average cost of the trajectory of optimal operation and thus shows that in this sense the closed loop also performs well on long time horizons.

\section{NUMERICAL EXAMPLE}

We conclude this paper by giving a simple example that demonstrates the kind of problem we can tackle with the economic MPC algorithm.

Example 1: Consider the system

$$
x(k+1)=x(k)+u(k)+w(k)
$$

where $w(k)=-2 \sin \left(\frac{k \pi}{12}\right)+a_{k}$ and $\left(a_{k}\right)_{k \in \mathbb{N}_{0}} \subset\left[-\frac{1}{4}, \frac{1}{4}\right]$ is a sequence of random numbers. Let $\mathbb{X}(k)=[-2,2]$, for $k \in\left[24 j, 24 j+12\left[\right.\right.$, and $\mathbb{X}(k)=\left[-\frac{1}{2}, \frac{1}{2}\right]$, for $k \in[24 j+$ $12,24(j+1)\left[, j \in \mathbb{N}_{0}\right.$, and let $\mathbb{U}(k)=[-3,3], k \in \mathbb{N}_{0}$. We thus use the stage cost $\ell(k, x, u)=u^{2}$. The goal in this example is to keep the state $x$ within the set $\mathbb{X}(k)$ with minimal control effort.

The setting could be interpreted as keeping the temperature of a room within a certain range while spending as little energy as possible. In this setting, the sequence $w(k)$ would correspond to the time-varying outside temperature, which can be measured.

Numerical evidence from simulations suggests that the system satisfies the turnpike property and that there exists an overtaking optimal trajectory $\left(x^{*}, u^{*}\right)$. However, we cannot compute this trajectory analytically.

The continuity assumption of the optimal value functions is also satisfied for this example as outlined in the following: Consider $x_{1}:=x^{*}(k)$ and the corresponding optimal control sequence $u_{N, x_{1}}^{*}$. Let $x \in \mathcal{B}_{\varepsilon}\left(x_{1}\right) \cap \mathbb{X}(k)$ and construct a control sequence $\tilde{u} \in U^{N}$ by

$$
\tilde{u}(j):= \begin{cases}x_{1}-x+u_{N, x_{1}}^{*}(0), & j=0 \\ u_{N, x_{1}}^{*}(j), & j=1, \ldots, N-1 .\end{cases}
$$

By construction the trajectories $x_{\tilde{u}}$ and $x_{u_{N, x_{1}}^{*}}$ coincide for all except the first time instant. Thus we have

$$
\begin{aligned}
& \hat{V}_{N}(k, x)-\hat{V}_{N}\left(k, x_{1}\right) \leq J_{N}(k, x, \tilde{u})-J_{N}\left(k, x_{1}, u_{N, x_{1}}^{*}\right) \\
& =(\underbrace{x_{1}-x}_{=: r}+u_{N, x_{1}}^{*}(0))^{2}-u_{N, x_{1}}^{*}(0)^{2}=r^{2}+2 r u_{N, x_{1}}^{*}(0) \\
& \leq r^{2}+6|r|:=\gamma_{V}(N, r)
\end{aligned}
$$



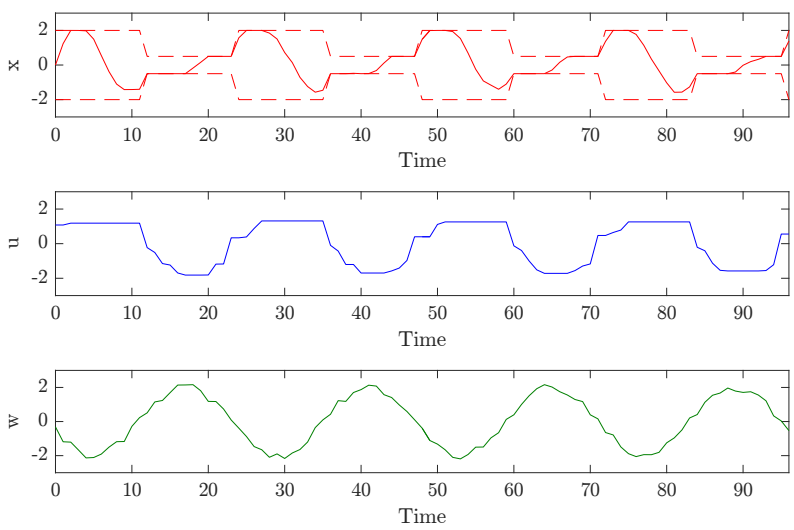

Fig. 2. MPC solution starting from the initial state $x(0)=0$ for a horizon length of $N=10$.

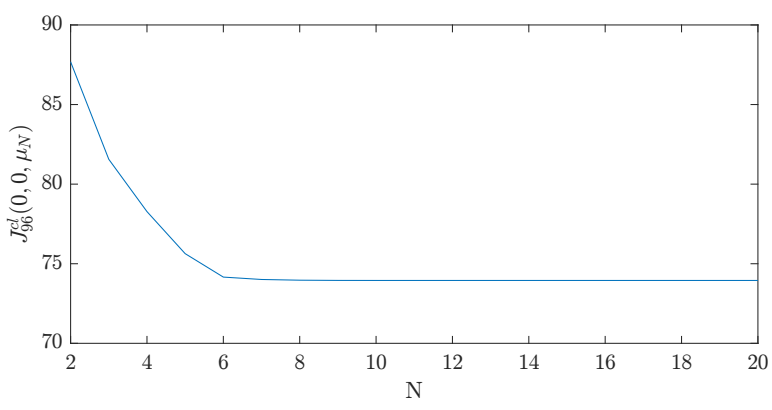

Fig. 3. Closed loop cost for the example.

using that $u_{N, x_{1}}^{*}(0)$ is uniformly bounded for all $N \in \mathbb{N}$. Observing that $\gamma_{V}(N, r) \rightarrow 0$ for $r \rightarrow 0$ yields the desired continuity. The continuity of $\hat{V}_{\infty}$ follows similarly.

Figure 2 shows the closed loop solution of the economic MPC algorithm, as well as the corresponding control sequence and the disturbance $w$. Obviously the state remains within the constraints.

In Figure 3 one can see the closed loop cost $J_{96}^{c l}\left(0,0, \mu_{N}\right)$ evaluated along the MPC solution trajectory for different horizon lengths $N$. As the horizon increases the cost quickly converges to some value, according to our theory to the (unknown) value of the initial piece of the infinite horizon optimal trajectory.

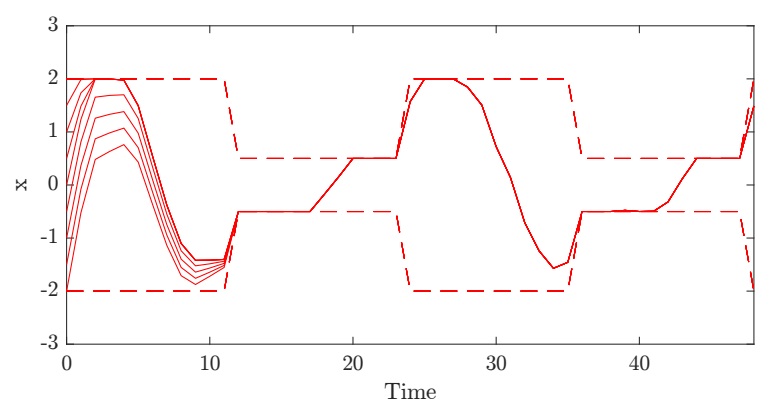

Fig. 4. MPC closed loop trajectories for different initial values of the system using a horizon of $N=10$.
Finally, in Figure 4 the MPC closed loops for different initial values are shown. We see that all solutions converge towards a single unique trajectory, which we conjecture to be the one at which the system is optimally operated.

\section{CONCLUSION AND OUTLOOK}

In this paper we presented performance estimates for economic model predictive control of time-varying systems. Under turnpike and continuity assumptions we could prove that the cost of the MPC closed loop approximates the cost of an infinite horizon optimal trajectory. The results were also illustrated with a numerical example.

As in the case of optimal equilibria, we conjecture that our key assumptions, turnpike and continuity of the optimal value function, hold under dissipativity and controllability conditions of the system. This will be addressed in future work. We will also investigate the convergence of the MPC closed loop towards the trajectory of optimal operation suggested by our numerical results in Figure 4.

\section{ACKNOWLEDGEMENTS}

The authors wish to thank Matthias Müller for his valuable input during discussion for this paper.

\section{REFERENCES}

[1] R. Amrit, J. B. Rawlings, and D. Angeli. Economic optimization using model predictive control with a terminal cost. Annual Rev. Control, 35:178-186, 2011.

[2] D. Angeli, R. Amrit, and J. B. Rawlings. On average performance and stability of economic model predictive control. IEEE Trans. Autom. Control, 57(7):1615-1626, 2012.

[3] D. P. Bertsekas. Dynamic programming and optimal control, volume 1. Athena Scientific Belmont, MA, 1995.

[4] J. Blot and N. Hayek. Infinite-horizon optimal control in the discretetime framework. Springer, 2014.

[5] M. Ellis, J. Liu, and P. D. Christofides. Economic Model Predictive Control. Theory, Formulations and Chemical Process Applications. Springer, 2017.

[6] D. Gale. On optimal development in a multi-sector economy. Rev Econ. Studies, 34(1):1-18, 1967.

[7] L. Grüne. Economic receding horizon control without terminal constraints. Automatica, 49(3):725-734, 2013.

[8] L. Grüne. Approximation properties of receding horizon optimal control. Jahresber. DMV, 118(1):3-37, 2016.

[9] L. Grüne and J. Pannek. Nonlinear Model Predictive Control. Theory and Algorithms. Springer, second edition, 2017.

[10] L. Grüne and M. Stieler. Asymptotic stability and transient optimality of economic mpc without terminal conditions. J. Proc. Control, 24(8):1187-1196, 2014.

[11] C. M. Kellett. A compendium of comparison function results. Math Control, Sign. Syst., 26(3):339-374, 2014.

[12] M. A. Müller, D. Angeli, and F. Allgöwer. On convergence of averagely constrained economic MPC and necessity of dissipativity for optimal steady-state operation. In Proceedings of the American Control Conference - ACC 2013, pages 3141-3146, Washington, DC, USA, 2013.

[13] M. A. Müller and L. Grüne. Economic model predictive control without terminal constraints for optimal periodic behavior. Automatica, 70:128-139, 2016

[14] M. Zanon, S. Gros, and M. Diehl. A Lyapunov function for periodic economic optimizing model predictive control. In Proceedings of the 52nd IEEE Conference on Decision and Control - CDC2013, pages 5107-5112, Florence, Italy, 2013.

[15] M. Zanon, L. Grüne, and M. Diehl. Periodic optimal control, dissipativity and MPC. IEEE Trans. Auto. Cont., 2017. To appear. Online version available via DOI:10.1109/TAC.2016.2601881. 